\title{
P62 Estimation of Wave Intensity in Humans Using only Pressure Waveforms and Reservoir Analysis
}

\author{
Alun Hughes ${ }^{1,2, *}$, Kim Parker $^{3}$, Nish Chaturvedi ${ }^{1,2}$, Chloe Park $^{1}$ \\ ${ }^{1}$ Department of Population Science \& Experimental Medicine, Institute of Cardiovascular Science, UCL, London, UK \\ ${ }^{2} M R C$ Unit for Lifelong Health and Aging@UCL, London, UK \\ ${ }^{3}$ Department of Bioengineering, Imperial College London, UK
}

\section{ABSTRACT}

Background: Measurement of wave intensity (WI) requires simultaneous or quasi-simultaneous measurement of pressure and flow limiting its use. Previous work in dogs [1] and humans [2] has shown that the excess pressure waveforms calculated using reservoir analysis correspond closely with aortic flow waveforms. This offers a potential method to estimate WI using only pressure waveforms (pWI). We investigated the feasibility of this approach and agreement with established methods.

Methods: 262 participants $(68.3(\mathrm{SD}=5.5) ; 74 \%$ male) without aortic stenosis or heart failure were recruited from a UK-based longitudinal study, Southall and Brent Revisited. Central pressure waveforms, aortic flow velocity and carotid WI were measured using tonometry (Sphygmocor, AtCor), echocardiography (iE33, Philips) and ultrasonography (SSD-5500, Aloka) respectively. Reservoir analysis was performed as previously described [2] and excess pressure waveforms were calibrated to flow velocity assuming a peak velocity of $1 \mathrm{~m} / \mathrm{s}$. Method agreement was assessed as mean difference (MD), limits of agreement (LOA) and concordance coefficient (CC).

Results: Analysis failed in 9 individuals; results for those with analysable data are shown in Table 1. Aortic pWI was higher than aortic WI but showed good concordance $(\log \mathrm{W} 1 \mathrm{MD}(\mathrm{LOA})=-0.41(-0.73,-0.09) \mathrm{CC}=0.7 ; \log 2 \mathrm{MD}(\mathrm{LOA})=-0.41(-0.73$, $-0.09)$; $C C=0.7)$. Agreement of pWI with carotid WI showed no bias and concordance was fair to poor $(\log 1: \mathrm{MD}(\mathrm{LOA})=$ $-0.16(-1.30,0.99) \mathrm{CC}=0.3 ; \log \mathrm{W} 2: \mathrm{MD}(\mathrm{LOA})=-0.02(-1.23,1.2) ; \mathrm{CC}=0.1)$.

Conclusion: Estimation of aortic WI from pressure waveforms using reservoir analysis is feasible.

Table 1 Results

\begin{tabular}{lcccc}
\hline Variables & $\mathbf{N}$ & Median/(\%) & p25 & p75 \\
\hline Age, y & 207 & 67.9 & 63.6 & 71.9 \\
BMI, kg/m ${ }^{2}$ & 207 & 26.6 & 24.1 & 30.1 \\
Systolic BP, mmHg & 207 & 139 & 126 & 148 \\
Diastolic BP, mmHg & 207 & 76 & 70 & 82 \\
Heart rate, bpm & 207 & 66 & 59 & 74.5 \\
Male sex, \% & 150 & 72.5 & & \\
Ethnicity & & & & \\
$\quad$ European & 88 & $(42.5 \%)$ & & \\
South Asian & 75 & $(36.2 \%)$ & & \\
African Caribbean & 44 & $(21.3 \%)$ & & \\
Current smoker & 18 & $(8.8 \%)$ & & \\
Diabetes & 65 & $(31.4 \%)$ & & \\
Hypertension & 130 & $(62.8 \%)$ & & \\
Aorta & & & & \\
$\quad$ W1, mmHg.m.s ${ }^{-3}$ & 207 & 7103 & 5041 & \\
W2, mmHg.m.s ${ }^{-3}$ & 207 & 1637 & 1147 & 2518 \\
pW1, mmHg.m.s s $^{-3}$ & 207 & 10,526 & 7677 & 14,336 \\
pW2, mmHg.m.s ${ }^{-3}$ & 207 & 2491 & 1779 & 3560 \\
time W1 to W2, s & 207 & 0.25 & 0.23 & 0.27 \\
Peak velocity, m/s & 207 & 1.34 & 1.2 & 1.46 \\
\hline
\end{tabular}


Table 1 Results

\begin{tabular}{lcccc}
\hline Variables & $\boldsymbol{N}$ & Median/(\%) & p25 & p75 \\
\hline Carotid artery & & & & \\
W1, mmHg.m.s $\mathrm{s}^{-3}$ & 207 & 8714 & 6550 & 12,883 \\
W2, mmHg.m.s ${ }^{-3}$ & 207 & 2327 & 1471 & 3227 \\
time W1 to W2, s & 207 & 0.29 & 0.27 & 0.31 \\
Peak velocity, m/s & 207 & 1.10 & 0.92 & 1.30 \\
\hline
\end{tabular}

BMI, body mass index; $\mathrm{BP}$, blood pressure; $\mathrm{p} 25,25$ th centile; $\mathrm{p} 75,75$ th centile; $\mathrm{pW}$, peak intensity of initial forward compression wave (W1) estimated using pressure only; pW2, peak intensity of initial forward compression wave (W2) estimated using pressure only; W1, peak intensity of initial forward compression wave (W1) calculated using aortic velocity; W2, peak intensity of initial forward compression wave (W2) calculated using aortic velocity.

\section{REFERENCES}

[1] Wang, et al. Am J Physiol Heart Circ Physiol 2003;284:H1358-H68.

[2] Michail, et al. Physiol Meas 2018;39:064006.

(c) 2019 Association for Research into Arterial Structure and Physiology. Publishing services by Atlantis Press International B.V. This is an open access article distributed under the CC BY-NC 4.0 license (http://creativecommons.org/licenses/by-nc/4.0/). 